\title{
Rehabilitation vor Pflege
}

\section{Die Teilhabe pflegebedürftiger Menschen ist umfassend geregelt}

\author{
Harry Fuchs
}

Harry Fuchs war bis 1995 Projektleiter im Ministerium für Arbeit, Soziales und Gesundheit Rheinland-Pfalz für die Reform des öffentlichen

Gesundheitsdienstes sowie der Behörden und Dienststellen der Sozialverwaltung des Landes. Seither ist er beurlaubt für wissenschaftliche Tätigkeiten in der Berliner Humboldt-Universität. Zudem ist er als Sachverständiger in den Bereichen Gesundheitswesen, Rehabilitation, Pflegeversicherung, Alterssicherung und Finanzierung von Sozialleistungssystemen tätig. Internet http://www.harry-fuchs.de
Wichtige gesetzliche Bestimmungen für pflegebedürftige Menschen finden sich nicht nur in der sozialen Pflegeversicherung (SGB XI); auch das Gesetz über die Rehabilitation und Teilhabe behinderter Menschen (SGB IX) ist für sie von großer Bedeutung. Ziel ist danach nicht nur die gesundheitliche Versorgung von Menschen mit einer Behinderung - zu denen auch Pflegebedürftige zu zäblen sind -, sondern vor allem ibre Selbstbestimmung und ibre gleichberechtigte Teilhabe am Leben in der Gesellschaft zu fördern.

Das am 1. Juli 2001 in Kraft getretene Neunte Sozialgesetzbuch (SGB IX) (1) fasst das Recht der Rehabilitation und Teilhabe behinderter Menschen in einem Gesetz zusammen, das nach seiner Begründung - wie die Sozialgesetzbücher I, IV und X - übergreifendes Recht und deswegen auch im Geltungsbereich des Pflegeversicherungsgesetzes (SGB XI) anzuwenden ist, soweit dieses nicht ausdrücklich abweichende Bestimmungen enthält (2), was jedoch nicht der Fall ist.

\section{These: Pflegebedürftige Menschen sind auch behinderte Menschen im Sinne des SGB IX}

Ob jemand im Rechtssinne ein behinderter Mensch ist, wird nach $\mathbb{} 2$ SGB IX nicht mehr durch Art und Schwere einer Erkrankung definiert, sondern dadurch, dass als Folge einer Erkrankung eine Beeinträchtigung seiner Teilhabe am Leben in der Gesellschaft eingetreten ist oder droht. Dieser mit dem SGB IX vollzogene Paradigmenwechsel erfasst nach $\mathbb{2} 26$ Abs. 1 Nr. 1 SGB IX ausdrücklich auch alle chronischen Krankheiten.

Krankheit und Behinderung sind Ursache sowohl der Pflegebedürftigkeit, wie auch der Beeinträchtigung der Teilhabe am Leben in der Gesellschaft. Ist bei einem Menschen als Folge von Krankheit oder Behinderung bereits Pflegebedürftig- keit eingetreten, so ist er in der Regel auch in seiner Teilhabe am Leben in der Gesellschaft beeinträchtigt. Pflegebedürftigkeit und Teilhabebeeinträchtigung schließen sich nämlich nicht aus. Im Gegenteil, sie bedingen sich, weil sie auf gemeinsamen Ursachen basieren.

Auch pflegebedürftige Menschen sind deshalb durchweg behinderte Menschen im Sinne des $\ 2$ SGB IX; häufig sogar besonders schwer behinderte Menschen. Selbst wenn eine Teilhabebeeinträchtigung ausnahmsweise noch nicht eingetreten sein sollte, droht sie jedenfalls. $(3,4)$ Pflegebedürftige Menschen haben deshalb als behinderte Menschen mit Pflegebedarf sowohl Anspruch auf Leistungen zur Pflege nach dem SGB XI, als auch Anspruch auf Leistungen zur Teilhabe nach dem SGB IX. (5)

\section{These: Rehabilitation heißt nicht Fürsorge und Versorgung, sondern Ermöglichung von Teilhabe und Selbstbestimmung}

Seit dem Inkrafttreten des SGB IX haben die Leistungen zur Teilhabe und Rehabilitation nicht mehr nur die Aufgabe, den Erfolg einer Krankenbehandlung zu fördern und zu unterstützen. Sie haben vor allem die Aufgabe, die Selbstbestimmung und die gleichberechtigte Teilhabe am Leben in der Gesellschaft zu fördern, Benachteiligungen zu vermeiden oder ihnen entgegen zu wirken. Damit wird nicht nur dem im Grundgesetz verankerten Benachteiligungsverbot behinderter Menschen im Sozialrecht Geltung verschafft. Rehabilitation hilft danach, die Grundrechte zu verwirklichen. (6, 7) Im Mittelpunkt der Rehabilitation soll nicht mehr die Fürsorge und Versorgung behinderter Menschen stehen, sondern ihre selbstbestimmte Teilhabe am Leben in der Gesellschaft und die Beseitigung der Hindernisse, die ihrer Chancengleichheit entgegenstehen. 
Der Gesetzgeber hat das SGB IX an der International Classifikation of Functioning, Disability and Health (ICF) der Weltgesundheitsorganisation (WHO) orientiert, die Teilhabe als Einbezogensein in eine Lebenssituation definiert. Die ICF bietet eine gemeinsame Sprache für die Beschreibung der funktionellen Gesundheit und stellt dazu ein systematisches Verschlüsselungssystem für Gesundheitsinformationssysteme bereit. Die in der ICF enthaltene internationale Definition des Begriffs der Rehabilitation entfaltet sozial- und leistungsrechtlich in Deutschland keine unmittelbare Wirkung. Die ICF wird - im Gegensatz zur Verwendung im wissenschaftlichen Bereich - einschließlich ihres Verschlüsselungssystems sozial- und leistungsrechtlich beispielsweise für die Feststellung des individuellen Leistungsbedarfs in Deutschland nur im Kontext der Bestimmungen des nationalen Sozialrechts - hier des SGB IX - wirksam.

\section{These: Das Gesetz verpflichtet zu einem umfassenden Teilhabemanagement}

Die Leistungen zur Teilhabe und Rehabilitation sind nach dem Finalitätsprinzip, also ohne Rücksicht auf die Ursache der Behinderung, ausgerichtet am Bedarf zu erbringen. Das SGB IX verpflichtet die Träger der Rehabilitations- und Teilhabeleistungen deshalb zu einem umfassenden Teilhabemanagement in dessen Mittelpunkt die Bedarfsfeststellung steht.

Zunächst haben alle Rehabilitationsträger, die über Sozialleistungen wegen oder unter Berücksichtigung einer Behinderung oder drohenden Behinderung zu entscheiden haben oder diese erbringen, im Rahmen dieser Leistungsverfahren kraft Gesetzes zu prüfen, ob Leistungen zur Teilhabe (erforderlich und) voraussichtlich erfolgreich sind. Da es kaum vorstellbar ist, dass ein pflegebedürftiger Mensch nicht auch Leistungen zur Krankenbehandlung erhält, haben die Krankenkassen in diesem Zusammenhang zu prüfen, ob Rehabilitationsleistungen erfolgversprechend sein können und diese gegebenenfalls von Amts wegen einzuleiten. (8)

\10 SGB IX verpflichtet die Rehabilitationsträger, den individuellen Bedarf an Rehabilitations- und Teilhabeleistungen funktionsbezogen festzustellen. Die funktionsbezogene Feststellung des Leistungsbedarfs umfasst die Feststellung von Beeinträchtigungen in den neun Bereichen der Teilhabe nach der ICF. Die Träger sollen die Feststellung des individuellen Leistungsbedarfs auf der von ihnen in der Bundesarbeitsgemeinschaft für Rehabilitation (9) vereinbarten Gemeinsamen Empfehlung »Einheitliche Begutachtung « basieren. (10) Die Krankenkassen treffen ihre Feststellungen jedoch nach der Begutachtungsrichtlinie des Medizinischen Dienstes der Krankenkassen (11), die von der Gemeinsamen Empfehlung abweicht. Die Vorgehensweise reduziert die Ziele der medizinischen Rehabilitation für pflegebedürftige Menschen auf »alltagsrelevante Beeinträchtigungen der Aktivitäten « oder die mit $\ 14$ SGB XI verbundenen Anforderungen. Damit wird - ohne gesetzliche Legitimation - der umfassende, auf die Förderung der Selbstbestimmung und Teilhabe am Leben in der Gesellschaft ausgerichtete Reha-Auftrag des SGB IX unzulässig eingeschränkt. (12)

Die Rehabilitationsträger haben gemeinsame örtliche Servicestellen einzurichten (13), die neben Information und Beratung insbesondere die Zuständigkeit zu klären und die Entscheidungen des Rehabilitationsträgers so umfassend vorzubereiten haben, dass dieser unverzüglich entscheiden kann. Neben diesen und anderen Aufgaben hat der Gesetzgeber den Servicestellen ausdrücklich originäre Case-Management-Aufgaben zur unterstützenden Begleitung, Koordinierung und Vermittlung zugewiesen. (14) Die gemeinsamen Servicestellen haben die Pflegekassen bei drohender oder bestehender Pflegebedürftigkeit an der Beratung und Unterstützung zu beteiligen. (15)

Ist zur Feststellung des Rehabilitationsbedarfs ein Gutachten eines Sachverständigen erforderlich, nimmt dieser im Auftrag des Rehabilitationsträgers innerhalb von zwei Wochen nach Auftragserteilung eine umfassende sozialmedizinische, bei Bedarf auch psychologische Begutachtung vor. Dem Berechtigten sind dazu in der Regel drei möglichst wohnortnahe Sachverständige zur Auswahl zu benennen. (16)

Der zuständige Rehabilitationsträger hat innerhalb von zwei Wochen nach Antragseingang seine Zuständigkeit festzustellen und innerhalb einer weiteren Woche (17) über den Leistungsantrag zu ent- scheiden. Hält sich der Rehabilitationsträger nicht für zuständig, hat er den Antrag unverzüglich - und das heißt: innerhalb von zwei Wochen - an den nach seiner Auffassung zuständigen Träger weiterzuleiten. Dieser hat dann immer auch wenn er letztlich doch nicht zuständig ist - innerhalb der genannten Fristen nach Antragseingang über die Leistung zu entscheiden, soweit er für die beantragte Leistungsart Träger im Sinne der $\mathbb{S} \mathbb{S} 5,6$ SGB IX ist. (18)

Neben und unabhängig vom Teilhabeund Versorgungsmanagement des SGB IX verpflichtet die Gesundheitsreform 2007 (19) die Krankenhäuser in $\mathbb{} 11$ Abs. 4 SGB V zur Einrichtung eines Entlassungsmanagements zur Gewährleistung des nahtlosen Übergangs von der Krankenhausbehandlung in die ambulante Versorgung, zur Rehabilitation oder Pflege. (20) Nach dem Pflegeweiterentwicklungsgesetz (21) sollen zudem Pflegestützpunkte eingerichtet werden, deren Aufgabe ebenfalls in der Organisation und Koordination aller für die wohnortnahe Versorgung und Betreuung pflegebedürftiger Menschen in Betracht kommenden gesundheitsfördernden, präventiven, kurativen, rehabilitativen und sonstigen medizinischen sowie sozialen Hilfs- und Unterstützungsangebote einschl. der Hilfestellung bei der Inanspruchnahme der Leistungen sein soll.

\section{These: Für Rehabilitations- und Teilhabeleistungen ist immer ein Träger zuständig}

Die Pflegekassen sind keine Träger von Rehabilitations- und Teilhabeleistungen. Dies sind für Leistungen der medizinischen Rehabilitation an pflegebedürftigen Menschen in der Regel die Krankenkassen. (22) Träger der Leistungen zur Teilhabe am Leben sind für pflegebedürftige Menschen in der Regel die Träger der Sozialhilfe, die durch das SGB IX für die Leistungen nach $\mathbb{S} \mathbb{S} 55$ SGB IX in den Kreis der Rehabilitationsträger einbezogen wurden. Doch wie auch immer: In Deutschland ist für Rehabilitations- und Teilhabeleistungen für behinderte, chronisch kranke und pflegebedürftige Menschen in jedem Fall ein Träger zuständig und leistungsverpflichtet.

Die Pflegekassen haben im Einzelfall bei Leistungsgewährung im Zusammen- 
hang mit Nachuntersuchungen - zu prüfen, ob medizinische Leistungen zur Rehabilitation geeignet und zumutbar sind und bei der Einleitung und Ausführung solcher Leistungen eng mit dem Rehabilitationsträger zusammenzuarbeiten. (23) Stellt der Medizinische Dienst der Krankenkassen einen entsprechenden Bedarf fest, haben sie dem zuständigen Rehabilitationsträger eine Mitteilung zuzuleiten, die bei Einwilligung des Versicherten zugleich als Antragstellung gilt. Die Pflegekasse ist über die Entscheidung des Rehabilitationsträgers zu informieren und hat in einem angemessenen zeitlichen Abstand zu prüfen, ob entsprechende Maßnahmen durchgeführt worden sind. (24)

Wird der Rehabilitationsträger nicht innerhalb von vier Wochen tätig, erbringt die Pflegekasse nach vorheriger Unterrichtung und Hinweis auf die Eilbedürftigkeit selber die Rehabilitationsleistungen vorläufig , wovon in der Praxis bisher faktisch kein Gebrauch gemacht wird.

\section{These: Die Leistungs- ansprüche zur Teilhabe und Rehabilitation finden sich in zahlreichen Sozialgesetzen}

Der Gesetzgeber geht von einer weitgehenden Einheitlichkeit des Leistungsrechts aus, die von den Trägern durch Koordination, Kooperation und Konvergenz zu gewährleisten ist. (26) Der behinderte, pflegebedürftige und chronisch kranke Mensch soll - völlig losgelöst von der Zuständigkeit eines Trägers und der Ursache seines individuellen Rehabilitationsbedarfs - aus gegebenem Anlass von jedem zuständigen Rehabilitationsträger die nach Art, Umfang sowie Strukturund Prozessqualität gleich wirksame und bedarfsgerechte Rehabilitations- und Teilhabeleistung erhalten.

Die Leistungen zur Teilhabe für pflegebedürftige Menschen werden nach $\mathbb{} 5$ SGB IX in folgenden Leistungsgruppen (27) erbracht

- Leistungen zur medizinischen Rehabilitation $(\mathbb{S} 26$ bis 32$)$

- unterhaltssichernde und ergänze Leistungen ( $\mathbb{S} 44$ bis 54)

- Leistungen zur Teilhabe am Leben in der Gemeinschaft ( $\mathbb{S} 55$ bis 59).

Die Leistungen werden zur Erreichung folgender gesetzlich vorgegebener Ziele gewährt a) die Behinderung abzuwenden, zu beseitigen, zu mindern, ihre Verschlimmerung zu verhüten oder ihre Folgen zu mildern

b) Einschränkungen der Erwerbsfähigkeit oder Pflegebedürftigkeit zu vermeiden, zu überwinden, zu mindern oder eine Verschlimmerung zu verhüten sowie den vorzeitigen Bezug anderer Sozialleistungen zu vermeiden oder laufende Sozialleistungen zu mindern

c) die persönliche Entwicklung ganzheitlich zu fördern und die Teilhabe am Leben in der Gesellschaft sowie eine möglichst selbstständige und selbstbestimmte Lebensführung $\mathrm{zu}$ ermöglichen oder zu erleichtern.

Das letzte Ziel ist gerade für die medizinische Rehabilitation pflegebedürftiger Menschen unabdingbare Leistungsvoraussetzung. Ob solche Ziele mit Leistungen zur Teilhabe erreichbar erscheinen, ist mit der Feststellung des individuellen funktionsbezogenen Leistungsbedarfs nach $\mathbb{S}$ 10 SGB IX zu prüfen (Teilhabeprognose).

Sozialrechtlich gesehen reduziert sich der Anspruch auf soziale Hilfen und Leistungen für pflegebedürftige und/oder behinderte Menschen nicht auf die Leistungen des SGB XI, sondern kann inter- und multidisziplinär durch eine Vielzahl von Gesetzen des Sozialrechts (SGB V, VII, VIII, IX, XI und XII; BVG u. a.) weit über die Leistungen des SGB XI hinaus begründet sein, je nachdem, welche Art und Ausprägung der Teilhabebeeinträchtigung den Hilfebedarf verursacht (z. B. Beeinträchtigung der körperlichen und seelischen Integrität - SGB V, VII, BVG u. SGB XI; Beeinträchtigung der sozialen Integrität SGB VII, IX und XII, BVG; Beeinträchtigung der Aktivitäten und Leistungen SGB VII, VIII, IX, XI und XII, BVG usw.).

\section{These: Der Rehabilitations- träger ist selbst für Leistungs- inhalt und Qualität verantwortlich}

Die Rehabilitationsträger entscheiden auf der Grundlage des Sachverständigengutachtens oder gleichwertiger Unterlagen mit Aussagen zu Art und Umfang der bei dem Berechtigen vorliegenden Teilhabebeeinträchtigung nach den Bestimmungen des für sie jeweils geltenden Sozialgesetzbuches über Art, Dauer, Umfang, Beginn und Durchführung der Leistung sowie die Rehabilitationseinrichtung (28) und erlassen darüber einen Verwaltungsakt (29).

Im Gegensatz zur Krankenversorgung nach dem SGB V, bei der die Verantwortung für die Leistungsausführung bei den Leistungserbringern liegt, sieht das SGB IX ausdrücklich vor, dass der zuständige Rehabilitationsträger die Leistungen zur Teilhabe selbst ausführt (30) und für Leistungsinhalt und Qualität verantwortlich bleibt. Die Auswahl der Rehabilitationseinrichtung hat danach zu erfolgen, welcher Dienst oder welche Einrichtung die Leistung in der am besten geeigneten Form ausführt, bezogen auf die Erreichung der angestrebten Teilhabeziele mit einer besonders wirksamen und wirtschaftlichen Struktur- und Prozessqualität. (31) Die Rehabilitationsträger teilen dazu der Rehabilitationseinrichtung die mit der Rehabilitationsleistung anzustrebenden Ziele als Maßstab für die anzustrebende Ergebnisqualität mit.

\section{These: Geriatrische Rehabilitation umfasst alle geeigneten Rehabilitationsleistungen}

Sozialrechtlich wurde der sozialpolitische Grundsatz »Rehabilitation vor Pflege « bereits durch die Gesundheitsreform 2000 (32) als ausdrückliche Aufgabe der Rehabilitation der Krankenversicherung mit der Zielsetzung der Vermeidung oder Minderung von Pflegebedürftigkeit umgesetzt. Geriatrische Rehabilitation umfasst die Rehabilitationsleistungen, die bei drohender oder bereits eingetretener Pflegebedürftigkeit geeignet sind, die als Folge der im Fachgebiet Geriatrie behandelten Krankheiten eingetretenen Beeinträchtigungen der betroffenen Menschen bei ihrer Teilhabe am Leben in der Gesellschaft zu behandeln. Geriatrische Rehabilitation ist im Kontext des Rehabilitationsrechts keine besondere Methode der Behandlung von Alterskrankheiten, sondern eine Leistungsform der medizinischen Rehabilitation, auf die in der Gesetzlichen Krankenversicherung bereits seit der Gesundheitsreform 2000 ein Rechtsanspruch besteht. Sie kann - wie alle Leistungen zur medizinischen Rehabilitation der Gesetzlichen Krankenversicherung - in ambulanter oder stationärer Form erbracht werden. (33) 
- Ambulante Rehabilitation soll zunächst in stationären Rehabilitationseinrichtungen, für die ein Versorgungsvertrag nach $\mathbb{} 111$ SGB V besteht (34), ausgeführt werden. Soweit dies für eine bedarfsgerechte, leistungsfähige und wirtschaftliche Versorgung erforderlich ist, kann sie mit gleicher Qualität und Wirksamkeit in wohnortnahen Einrichtungen erbracht werden, mit denen nach der Gesundheitsreform 2007 ein Vertrag nach $\mathbb{S} 40$ SGB V einzugehen ist. Die Spitzenverbände haben die Anforderungen an die Struktur- und Prozessqualität der ambulanten geriatrischen Rehabilitationseinrichtungen in Rahmenempfehlungen festgelegt (35), die noch nicht an den weitergehenden Anforderungen des SGB IX orientiert sind.

- Mit der Gesundheitsreform 2007 wurde klargestellt, dass die ambulante Rehabilitation »durch « wohnortnahe Einrichtungen erbracht wird, und dass ambulante Rehabilitation auch in stationären Pflegeeinrichtungen (36) zu erbringen sind. (37) Im Übrigen kann unter Beachtung des SGB IX, das ausdrücklich die Ausführung der Leistungen durch Rehabilitationsdienste unabhängig von der Art der Wohnung oder Unterbringung vorsieht, kein Zweifel daran bestehen, dass die ambulante mobile Rehabilitation auch in Behinderteneinrichtungen $\mathrm{zu}$ erbringen ist.

- Leistungen der geriatrischen Rehabilitation können grundsätzlich auch im Rahmen eines Persönlichen Budgets nach $\mathbb{S} 17$ Abs. 3 SGB IX ausgeführt werden. (38) Budgetfähig sind nämlich alle Leistungen der Gesetzlichen Krankenversicherung, soweit sie sich auf alltägliche und regelmäßig wiederkehrende Bedarfe beziehen. (39) Danach können im Rahmen von Persönlichen Budgets nach $\mathbb{} 17$ SGB IX Leistungen der Akutversorgung (z. B. Hauspflege) mit denen der ambulanten geriatrischen Rehabilitation, Leistungen der Pflegeversicherung oder Leistungen der Eingliederungshilfe kombiniert und aus einer Hand, also durch einen Leistungserbringer erbracht werden. (40) Während mit dem Personenbezogenen Budget nach $\mathbb{\int} 8$ Abs. 3 SGB XI im Rahmen von Modellvorhaben »neue qualitätsgesicherte Versorgungsformen « nur des SGB XI für pflegebedürftige Menschen erprobt wurden, ge- währt $\int 17$ SGB IX einen rechts- und trägerübergreifenden Anspruch auf Ausführung verschiedener Sozialleistungen aus unterschiedlichen Sozialgesetzen durch einen der leistungsverpflichteten Träger.

- Als Frührehabilitation nach $\$ 39$ Abs. 1 Satz 3 SGB V Nach $\mathbb{\$} 39$ Abs. 1 Satz 3
- Eine weitere Form zur Überwindung von Schnittstellenproblemen und zur Kombination unterschiedlicher Leistungen nach den verschiedenen Sozialgesetzbüchern stellen an der Schnittstelle von Akutversorgung, Rehabilitation und Pflege die Verträge zur integrierten Versorgung nach $\mathbb{S} \mathbb{S} 140 \mathrm{a}$

\section{»Beim Persönlichen Budget wird der Leistungsberechtigte zum Manager seiner Versorgung"}

SGB V umfasst die akutstationäre Behandlung im Krankenhaus auch die im Einzelfall erforderlichen und zum frühestmöglichen Zeitpunkt einsetzenden Leistungen der Frührehabilitation. Bei den »Leistungen zur Frührehabilitation « handelt es sich nicht um eine besondere Leistungsart der medizinischen Rehabilitation im Sinne des $\$ 26$ Abs. 2 und 3 SGB IX. Die Bezeichnung "Leistungen zur Frührehabilitation « beschreibt eine bestimmte Zeitphase der Akutversorgung, in der nach $\$ 2$ a SGB V den besonderen Belangen behinderter und chronisch kranker Menschen während der Krankenhandlung dadurch Rechnung zu tragen ist, dass neben akutmedizinischen Interventionen bereits parallel rehabilitative Methoden mit der Zielsetzung des SGB IX (41) einzusetzen sind. Die Leistungen zur geriatrischen Frührehabilitation nach \39 SGB V sind danach unmittelbarer Bestandteil der Krankenhausversorgung. Erfordern die Behandlungsziele keine weitere stationäre Akutversorgung mehr, darf die Krankenhausbehandlung nicht fortgesetzt werden. Ist die Frührehabilitation zu diesem Zeitpunkt nicht abgeschlossen, wird sie gegebenenfalls während der anschließenden Rehabilitationsversorgung fortgesetzt. Die im Krankenhaus für die Leistungen zur geriatrischen Frührehabilitation anfallenden Kosten sind Gegenstand der Krankenhausvergütung. Die Anforderungen, die das Krankenhaus erfüllen muss, um den Aufwand im Rahmen der Fallpauschalen vergütet zu erhalten, sind im Operationen- und Prozedurenschlüssel (OPS) zum Fallpauschalenkatalog 2004 (42) beschrieben. ff. SGB V dar, an denen nach $\mathbb{1} 140 \mathrm{~b}$ Abs. 1 SGB V (43) in Verbindung mit $\mathbb{S}$ 92b SGB XI nunmehr auch Pflegekassen und nach dem SGB XI zugelassene Pflegeeinrichtungen beteiligt sein können. Bei der Einbeziehung pflegerischer Leistungen in die integrierte Versorgung darf - vergleichbar der Regelung für die Leistungen der Krankenbehandlung (44) - zwar von den Vorschriften des SGB XI über die Leistungsinhalte und deren Ausgestaltung (45), dem Pflegesatzverfahren (46) und den Grundsätzen der Vergütungsregelungen (47), nicht aber von den Regelungen über die Leistungsvoraussetzungen, deren Feststellung und die Höhe der darauf basierenden Zuschüsse der Pflegekasse abgewichen werden.(48) Deshalb ist auch im Rahmen der integrierten Versorgung zunächst eine Feststellungs- und Einstufungsbegutachtung durch den Medizinischen Dienst der Krankenkassen erforderlich, bevor feststeht, welche Mittel der Pflegeversicherung im Rahmen der integrierten Versorgung verfügbar sind.

Für den Berechtigten besteht der wesentliche Unterschied zwischen der Leistungsvernetzung durch ein Persönliches Budget oder der integrierten Versorgung darin, dass es sich bei der integrierten Versorgung um einen Prozess zwischen Kostenträgern und Leistungserbringern handelt, an dem er nicht beteiligt ist und auf den er auch keinen Einfluss hat. Demgegenüber fördert das Persönliche Budget seine Selbstbestimmung, weil er damit weitgehend eigenverantwortlich entscheiden kann, wann er welche Leistungen, auf welche Weise und bei welchem Leistungserbringer durchführt - und er damit zum Manager seiner Versorgung wird. 


\section{Anmerkungen}

(1) Sozialgesetzbuch Neuntes Buch (SGB IX) Rehabilitation und Teilhabe behinderter Menschen vom 19.6.2001. BGBI I 2001; (27): 1046.

(2) $\$ 7$ Satz 1 SGB IX.

(3) Fuchs 2007c.

(4) $\ 2$ Abs. 2 Satz 2 SGB IX.

(5) Bundesbehindertenbeauftragte, Dezember 2006.

(6) $₫ 1$ SGB IX.

(7) Hans F. Zacher (2001), S. 1, 12.

(8) $\ 8$ SGB IX.

(9) Bundesarbeitsgemeinschaft für Rehabilitation, Frankfurt am Main, Internet http://www.bar-frankfurt.de

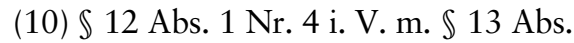
1 SGB IX.

(11) Begutachtungs-Richtlinie »Vorsorge und Rehabilitation " des Medizinischen Dienstes der Spitzenverbände der Krankenkassen e.V. (MDS) vom 28.10.2005.

(12) Vgl. dazu Bewertung der Richtlinie nach den Maßstäben des SGB IX, Internet http://www.harry-fuchs.de.

(13) $\mathbb{S}$ S22,23 SGB IX.

(14) $\ 22$ Abs. 1 Nr. 6-8 SGB IX.

(15) $\$ 22$ Abs. 1 Satz 4 SGB IX.

(16) $\ 14$ Abs. 5 SGB IX.

(17) Oder innerhalb von zwei Wochen nach Eingang eines erforderlichen Gutachtens.

(18) $\mathbb{1 4}$ Abs. 1 und 2 SGB IX.

(19) Gesetzes zur Stärkung des Wettbewerbs in der gesetzlichen Krankenversicherung (GKV-Wettbewerbsstärkungsgesetz - GKV-WSG). Bundestags-Drucksache 16/3100 vom 24.10.2006.

(20) Vgl. dazu ausführlich Fuchs, H., $2007 a$.

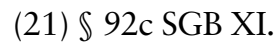

(22) In einigen Fällen können dies auch die Berufsgenossenschaften, die Versorgungsverwaltung, die Jugendämter oder im Rahmen der Eingliederungshilfe die Sozialämter sein.

(23) $\mathbb{3} 31$ Abs. 1 und 2 SGB XI.

(24) $\$ 31$ Abs. 3 SGB XI i.d.F. des PfWG.

(25) $\mathbb{3} 32$ SGB XI.

(26) Bundestags-Drucksache 14/5074, S. 100.

(27) Leistungen zur Teilhabe am Arbeitsleben $(\mathbb{S} \$ 33$ bis 43) kommen in der Regel nur bei jüngeren pflegebedürftigen Menschen in Betracht und werden hier nicht weiter vertieft.
(28) Z. B. $\iint 40$ Abs. 3 Satz 1 SGB V, 13 Abs. 1 Satz 1 SGB VI.

(29) $\ 31$ SGB X.

(30) $\$ 17$ Abs. 1 SGB IX.

(31) \ 19 Abs. 4 SGB IX.

(32) Gesetz zur Reform der gesetzlichen Krankenversicherung ab dem Jahr 2000 (GKV-Gesundheitsreformgesetz 2000 - GKV-GRG 2000) vom 22.12.1999. BGBl I 1999; (59): 2626.

(33) $\int 40$ Abs. 1 und 2 SGB V.

(34) $\int 40$ Abs. 1 SGB V.

(35) Rahmenempfehlungen zur ambulanten geriatrischen Rehabilitation vom 1.1.2004. In: Gerkens/Schliehe/ Steinke (Hrsg), 2006: 9-104.

(36) Im Sinne von $\mathbb{} 72$ Abs. 1 SGB XI. (37) $\ 40$ Abs. 1 Satz 2 SGB V.

(38) Die insoweit entgegen stehenden Empfehlungen der Spitzenverbände der Kranken- und Pflegekassen vom 28.6.2004 entsprechen nicht mehr dem geltenden Recht. Sie berücksich- tigen insbesondere nicht die am 1.7.2004 geänderte Rechtslage, nach der die »Regiefähigkeit der Leistungen « keine Voraussetzung mehr für die Budgetfähigkeit der Leistungen ist.

(39) $\mathbb{1 7}$ Abs. 2 Satz 4 SGB IX.

(40) Zum Persönlichen Budget nach $\mathbb{S}$ 17 SGB IX wird auf die diesbezüglichen Veröffentlichungen des Verfassers verwiesen, Internet http:// www.harry-fuchs.de.

(41) $\mathbb{S} \mathbb{S} 1,4$ Abs. 1 und 26 Abs. 1 SGB IX.

(42) Operationen- und Prozedurenschlüssel (OPS) zum Fallpauschalenkata$\log 2004$, Internet http://www.dimdi.de.

(42) In der Fassung des GKV-WSG.

(43) $\mathbb{1} 140$ b Abs. 4 SGB V.

(44) $\mathbb{} 75$ SGB XI.

(45) $\ 85$ SGB XI.

(46) $\ 89$ SGB XI.

(47) $\ 92$ b Abs. 2 Satz 2 und 3 SGB XI.

(48) \18 SGB XI.

\section{Literatur}

Beauftragte der Bundesregierung für die Belange behinderter Menschen, Dezember 2006, Arbeitskreis Teilhabeorientierte Pflege. Empfehlungen für eine Teilhabeorientierte Pflege, Internet http://www.behindertenbeauftragte.de.

Bihr/Fuchs/Krauskopf/Ritz (Hg.) SGB IX - Kommentar und Praxishandbuch. Sankt Augustin. Asgard 2006.

Fuchs, H. (2002): Einführung. In: SGB IX - Rehabilitation und Teilhabe behinderter Menschen. Beck-Texte im dtv, Nördlingen, 2. Auflage.

Fuchs, H. (2004) Medizinische Leistungen zur Rehabilitation und integrierte Versorgung Rehabilitation 2004; 43: 1-10.

Fuchs, H. (2006) Das Persönliche Budget. Sozialleistungen aus einer Hand. Betreuungsmanagement 2006; (2): $90 \mathrm{ff}$.

Fuchs, H. (2006) Die Gesundheitsreform 2006: Systemwechsel durch die Hintertür. Soziale Sicherheit 2006; (10): 342 ff.

Fuchs, H. (2007a) Anspruch auf Versorgungsmanagement für Menschen mit Pflegebedarf. Soziale Sicherheit 2007; (10): 338 ff.

Fuchs, H. (2007b) Geriatrische Rehabilitation aus dem Blickwinkel des Neunten Buches Sozialgesetzbuch (SGB IX) Rehabilitation 2007; 46: 296-309.

Fuchs, H. (2007c) Pflege und Rehabilitation in Igl/Naegele/Hamdorf (Hg.) Reform der Pflegeversicherung. Auswirkungen auf die Pflegebedürftigen und Pflegepersonen. LIT Verlag Hamburg 2007, 180-192.

Gerkens/Schliehe/Steinke (Hg.): Handbuch Rehabilitation und Vorsorge. Sankt Augustin: Asgard, 2006.

Medizinischer Dienst der Spitzenverbände der Krankenkassen (MDS). Begutachtungs-Richtlinie Vorsorge und Rehabilitation vom 28.10.2005. Essen: MDS, 2005, Internet http://www.mds-ev.org.

Zacher, Hans F. Der soziale Rechtsstaat in der Verantwortung für Menschen mit Behinderungen in: Gerhard Igl/Felix Welti, Die Verantwortung des sozialen Rechtsstaates für Personen mit Behinderung und für die Rehabilitation, 2001, S. 1, 12. 